\title{
Which green matters for whom? Greening and firm performance across age and size distribution of firms
}

\author{
Mili Shrivastava $\cdot$ Jagannadha Pawan Tamvada
}

Accepted: 30 June 2017 / Published online: 17 November 2017

C) The Author(s) 2017. This article is an open access publication
Keywords Green entrepreneurship · Green start-ups Sustainability · Types of greening · Greening framework · Age and size distribution of firms · Firm growth . Performance

\section{Introduction}

Sustainable development and the role of firms as agents of sustainable development is a topic of remarkable scholarly interest. Firms are typically considered as entities with sole motives of profit maximisation. Although a tradeoff between profit maximisation and sustainable business models was traditionally assumed, an emerging body of research suggests that firms can boost their performance while pursuing strategies that are considerate towards the environment (Hart and Ahuja 1996; Stefan and Paul 2008). A compelling stream of literature on sustainability has emerged that primarily focuses on how sustainable processes can be designed and developed for large established firms (King and Lenox 2001; Margolis and Walsh 2001; Stefan and Paul 2008). However, young and small firms can be agents for offering radical solutions to the challenges of sustainability, as they have an advantage in innovation (Acs and Audretsch 1990). In this context, Klewitz and Hansen (2014) provide a systematic review of sustainable innovation practices of small and mediumsized enterprises and suggest that they innovate differently.

The literature considers a wide range of greening initiatives of firms such as incorporating environmental management systems, obtaining compliance certifications, reducing emissions, and offering green products and services. However, there is no single framework that underpins the differences between these greening approaches and their impact on firm-level performance outcomes. We bridge this gap by developing a novel greening framework that consolidates these different greening initiatives using the lens of their tangibility and visibility to stakeholders.

J. P. Tamvada

Centre for India and Global Business, University of Cambridge, Cambridge, UK 
Fig. 1 Greening framework model

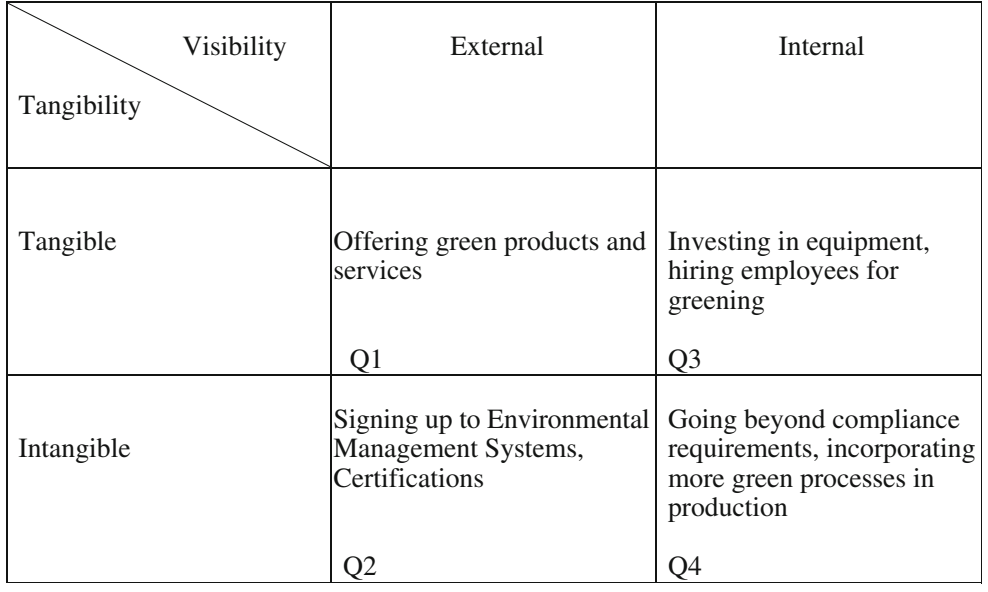

Furthermore, while several meta-analyses suggest that there is a positive relationship between green initiatives of firms and their performance (Margolis and Walsh 2001; Orlitzky et al. 2003; Allouche and Laroche 2006; Stefan and Paul 2008), little is known about how they differentially impact entrepreneurial young firms and mature incumbents or small and large firms. We bridge this important gap in the literature by linking different greening strategies with firm performance across the age and size distribution of firms. The empirical results based on a large-scale Eurobarometer database suggest that external greening activities have a positive impact on the performance of young firms and small firms. In contrast to this, internally perceived green processes play a significant role for middle-aged firms and large incumbent firms.

The paper makes several compelling contributions to the existing literature. Firstly, building on the input-output framework (Busch and Hoffmann 2011; Lannelongue et al. 2015), it presents a novel greening framework for contextualising greening by firms. Secondly, it provides the first insights into how different types of greening impact firm performance along the size and age distribution of firms. Thirdly, the large-scale database used for the empirical analysis includes firms from 38 different economies giving an opportunity to draw broad conclusions about the impact of greening on firm performance at an international scale.

The rest of the paper is organised as follows. Section 2 provides the theoretical background for the paper and develops the greening framework. The section presents the hypotheses linking greening initiatives and firm performance across the age and size distribution of firms. Section 3 discusses the database and the methodology used for the empirical analysis. Section 4 presents the empirical results. Section 5 provides a discussion of the results and concludes the paper.

\section{Theoretical background}

The link between environment and business has received increasing attention in scholarly literature (Bansal and Roth 2000). Although the question of whether it pays to be green has been addressed by numerous studies, it is far from conclusive. While traditionally it was assumed that addressing environmental concerns of firms' activities leads to increased costs for the firms (Coase 1960; Gray and Shadbegian 1993; Walley and Whitehead 1994), an emerging body of literature suggests that there may not be a contradiction between firms' discharging their environmental responsibilities and realising their economic goals (Porter and Van der Linde 1995; Nehrt 1996; Sharma and Vredenburg 1998; Dowell et al. 2000; Stefan and Paul 2008; Nishitani 2011).

In this context, Busch and Hoffmann (2011) suggest that both inputs and outputs relate to firms profits while Lannelongue et al. (2015) examine the impact of both green inputs and green outputs on firm performance. Their results suggest that green inputs, as well as green outputs, impact the financial performance of firms. We take this as a starting point for developing a new theoretical greening framework that characterises greening activities of firms as tangible or intangible activities that are visible externally or internally to the firm.

While some green activities and initiatives of firms are visible externally, others are internal processes that are mainly known to their management and employees. For instance, offering a green product or service in the 
marketplace is visible externally while hiring employees for green tasks is mainly known within the firm. The former is a tangible output and the latter is a tangible investment. Similarly, there are intangible green processes that the external world can be made aware of, for example, through environmental certifications, and intangible greening processes that are mainly perceived internally within the firm such as a firm adopting green processes for its production activities.

Figure 1 summarises the greening framework model. In the first quadrant (Q1), firms have tangible green outputs that are visible externally. In the second quadrant (Q2), firms signal to the external world about their intangible green processes using environmental management systems and certifications. In the third quadrant (Q3), firms have tangible investments such as employees hired for greening related tasks and, in the fourth quadrant (Q4), firms have intangible greening processes that are known mainly to their management and employees. ${ }^{1}$

While a majority of studies support the view that greening improves firm performance (Porter and Van der Linde 1995; Hart 1995; Hart and Ahuja 1996; Klassen and McLaughlin 1996; Sharma and Vredenburg 1998; Reinhardt 1998; Klassen and Whybark 1999; Dowell et al. 2000), few studies have suggested that it may have a negative effect (Cordeiro and Sarkis 1997; Sarkis and Cordeiro 2001). However, several meta-analyses confirm a positive link between environmental performance and firm performance (Margolis and Walsh 2001; Orlitzky et al. 2003; Allouche and Laroche 2006; Stefan and Paul 2008). Consistent with the findings of these meta-analyses, we hypothesise that all forms of greening have a positive impact on the firm's performance.

H1a: Externally tangible greening, as well as intangible greening, have a positive impact on firm performance

H1b: Internally tangible greening, as well as intangible greening, have a positive impact on firm performance

However, the 'who, how and when' it pays to be green are compelling questions that go beyond the basic question of whether it pays to be green. We use the greening framework conceptualised above to answer these

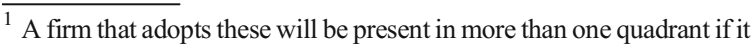
pursues more than one of the above greening strategies. For example, if the firm offers greens products in the market place and also has certifications about its environment initiatives, it has both tangible and intangible greening processes that are visible externally.
}

questions along the age and size distribution of firms. In this context, Hockerts and Wustenhagen (2010) suggest that a co-evolution of sustainability practices in entrepreneurial firms and large incumbent firms is more likely to lead to sustainability at industry level as Emerging Davids (small entrepreneurial firms) fail to reach broad mass markets but are more willing to pursue risky innovations (Coad et al. 2016) while the sustainability efforts of Greening Goliaths (large incumbents) are incremental and nonefficient (Hamschmidt and Dyllick 2001; Schaltegger 2002) as they face a real risk of cannibalising their market share (Nicholls and Opal 2005).

While greening may lead to competitive advantage for large incumbent firms (King and Lenox 2001; Esty and Winston 2009), entrepreneurial firms solve problems of environmental degradation by identifying and exploiting opportunities resulting from existing market failures (Cohen and Winn 2007; Dean and McMullen 2007) and do things that incumbent firms and institutions do not pursue (York and Venkataraman 2010). Consistent with this view, Stenzel and Frenzel (2008) show that incumbents reluctantly adopt renewable energy but a coevolutionary process between regulatory environment and technology strategies leads to their gradual adoption. Similarly, Wüstenhagen et al. (2003) suggest that while small firms initially offered green electricity in Switzerland, large incumbents slowly adopted the strategy of offering green products at different time points.

Thus, while incumbent firms may be hesitant to introduce products that cannibalise their existing products as this may impact their customer base, new entrants are more likely to identify niches and pursue them aggressively (Bhide 1994) while undertaking risky innovations (Coad et al. 2016). Young firms have a higher probability of innovation (Huergo and Jaumandreu 2004) and are more likely to have a greater $R \& D$ intensity than old firms when entering new markets (Czarnitzki and Kraft 2004) while having superior technical quality innovations (Balasubramanian and Lee 2008). Thus, green products and services offered by incumbent firms may not be radically different from their existing product portfolio, and producing these products may not have a dramatic impact on their performance. In contrast to this, the green products and services offered by new entrants are more likely to cater to an emerging trend or a niche market and are more likely to impact firm performance positively.

Furthermore, as Djupdal and Westhead (2015) suggest, externally intangible strategies such as certifications enable very young firms to address the liabilities of newness 
and the liabilities of smallness but these are not helpful as firms grow. Thus, externally tangible green initiatives such as offering green products or services and externally intangible green initiatives such as obtaining environmental certifications are likely to have a positive impact on the performance of young firms with the impact diminishing as firms mature. For these reasons, we hypothesise that external green signals have differential impacts across the age and size distribution of firms.

H2a: For entrepreneurial firms, external green strategies have a positive impact on firm performance $\mathrm{H} 2 \mathrm{~b}$ : As firms mature, external green strategies have a decreasing impact on firm performance

As firms undertake internally tangible greening initiatives such as hiring more green employees or internally intangible greening initiatives such as going beyond compliance requirements, they signal to their existing employees that they are taking proactive steps towards protecting the environment. This may positively impact their motivation (Temminck et al. 2015) and lead to an increased firm performance. These internal greening processes may result in cost-savings and efficiency gains as firms mature and, as Ford et al. (2014) suggest, firms may go beyond compliance requirements in pursuit of their competitive strategy. Consistent with these views, Chen et al. (2016) find that proactive engagement with environmental practices has a positive impact on the performance of large multinational firms, and Delmas et al. (2015) suggest that proactivity in going beyond environmental compliance requirements has a positive impact on firm performance in the long run. Furthermore, incumbents can innovate through processes (Hamschmidt and Dyllick 2001) or imitate rapidly successful sustainable innovations (Hockerts 2006). For these reasons, we hypothesise that tangible, as well as intangible internal greening, have a positive impact on firm performance across the age and size distribution of firms.

H3a: For entrepreneurial firms, internal green strategies have a positive impact on firm performance $\mathrm{H} 3 \mathrm{~b}$ : As firms mature, internal green strategies have a positive impact on firm performance

An important consideration is the potential of firms engaging in multiple greening strategies. For example, firms may pursue both tangible and intangible greening strategies or both internal and external greening strategies. While on the one hand pursuing multiple strategies may positively impact the firm, the multiplicity strategy can also increase the cost of greening for the firm. Thus, these contrasting effects together determine whether a firm experiences a net benefit from simultaneously pursuing multiple greening strategies.

\section{Methods}

\subsection{Data and variables}

We use a large-scale database commissioned by the European Commission, the Eurobarometer survey Eurobarometer (2012), for testing the hypotheses developed in Section 2. The data were collected through telephonic interviews from an international business register. Firms from 38 different countries were selected using a stratified sampling procedure. ${ }^{2}$ Although the original size of the sample is 13,167 firms, data of the dependent variable are available for 12,272 observations. Introducing the four greening strategies variables in the regression reduces the sample size to 9606 observations. Adding the firm size variables and the sampling weights brings the final sample size to 9236 observations. ${ }^{3}$

\subsubsection{Dependent variable}

Change in turnover The dependent variable is derived from a question in the database that asks if the firm's turnover has decreased, remained same or increased in the last 2 years. This is the only measure of firm performance available in the database. As Table 1 shows, $33.1 \%$ of the firms in the sample have experienced a decrease in turnover, while turnover has remained same for $26.6 \%$ of the firms and turnover has increased for $40 \%$ of the firms in the database.

\subsubsection{Independent variables}

The four different greening processes identified in the theory section are the main independent variables.

\footnotetext{
2 The list of countries included in the data collection process is given in the Appendix in Table 12.

${ }^{3}$ The mean values of the variables in the full sample and the final sample are not statistically different suggesting that exclusion is mostly random and not systematic.
} 
Table 1 Descriptive Statistics

\begin{tabular}{ll}
\hline Dependent variable: & \\
Turnover Decreased & $33.40 \%$ \\
Turnover Remained Same & $26.60 \%$ \\
Turnover Increased & $40.00 \%$ \\
& \\
Independent variables: & \\
GS1 Green Product or Service (Tangible-External) & $30.30 \%$ \\
GS2 Env. Mang. Sys (Intangible-External) & $38.60 \%$ \\
GS3 Green Employees Prop. (Tangible-Internal) & 0.16 \\
& $(0.46)$ \\
GS4 Beyond Compliance (Intangible-Internal) & $26.10 \%$ \\
Number of Green Strategies & 1.387 \\
Controls variables: & $(1.169)$ \\
Financial support & \\
Non-financial support & $9.15 \%$ \\
Consumers & $40.50 \%$ \\
Companies & $60.70 \%$ \\
Public Bodies & $70.20 \%$ \\
Age & $30.30 \%$ \\
Employees: 1 to 9 & 24.2 \\
Employees: 10-49 & $23.50 \%$ \\
Employees: 50-249 & 9236 \\
Employees > 250 & \\
Manufacturing & $23.59)$ \\
Retail & $32.50 \%$ \\
Services & $32.40 \%$ \\
Mining and other Industries & $18.10 \%$ \\
\hline & $27.55 \%$ \\
& $24.10 \%$ \\
& \\
\hline
\end{tabular}

Standard deviation in parentheses

GS1. Green Product or Service (Tangible-External Greening) This variable takes value 1 if the firm offers a green product or service to its customers. As Table 1 suggests, $30.3 \%$ of the firms in the database offer a green product or service.

GS2. Environmental Management System (Intangible-External Greening) If a firm has one of the formalised environmental management systems such as ISO14001, ISO14064, ISO16000 or others, the variable takes value 1 and 0 otherwise. Although the processes underlying these certifications may not be clear, firms declare these certifications to potential consumers and they are known externally. As Table 1 suggests $38.6 \%$ of firms in the database have environmental management systems in place.
GS3. Green Jobs Prop. (Tangible-Internal Greening) This variable is derived from a question in the survey that asks how many of the full-time employees of the firm work in green jobs some or all the time. According to the questionnaire, a 'green job is one that directly works with information, technologies, or materials that preserves or restores environmental quality. This requires specialised skills, knowledge, training, or experience.' As the number of green jobs cannot be viewed independently of the firm size, a new variable on the proportion of green jobs is constructed. ${ }^{4}$ The mean of this variable is 0.16 suggesting that, on an average, around $16 \%$ of full-time employees are engaged in green activities.

GS4. Beyond Compliance (Intangible-Internal Greening) The variable takes value 1 if a firm goes beyond complying with environmental legislations. As Table 1 suggests, $26.1 \%$ of the firms in the database proactively go beyond complying with environmental legislations.

\subsubsection{Control variables}

External support We control for the impact of external support as firms that receive external support are more likely to have an increased turnover. While half of the firms in the sample have received no external support, $9.15 \%$ have received financial support and $40.50 \%$ have received non-financial support. ${ }^{5}$

Market type The market type is controlled in the estimation as the market segment that firms supply to has an impact on their turnover. While $60.7 \%$ of the firm supply to consumers, $70.2 \%$ supply to companies and

\footnotetext{
${ }^{4}$ The exact number of employees is unavailable, and the employee sizes are coded in intervals such as 0 to 10 employees, 11-50 employees, 50-250 employees, 250-750 employees. We divide the number of green jobs by the midpoint of these intervals to derive the proportions.

${ }^{5}$ The question asked in the questionnaire is as follows: "Which type of external support does your company get in relation to its environmental actions?'. The answers to this question are from the following a. Public funding (grants or guarantees) b. Private funding from bank or investment companies c. Venture capital fund d. Advice or other non-financial assistance from public administration e. Advice or other non-financial assistance from private consulting and audit companies. f. Advice or other nonfinancial assistance from business associations. Firms that selected any one of the a. b. or c. options are classified as having received external financial support, and firms that selected any one of d. e. or f. are classified as having received external non-financial support.
} 
Table 2 Correlations between greening strategies

\begin{tabular}{lllll}
\hline & GS1 & GS2 & GS3 \\
\hline GS1. Green Product or Service (Tangible-External) & 1 & & GS4 \\
GS2. Env. Mang. Sys (Intangible-External) & $0.0689 * * *$ & 1 & 1 \\
GS3. Green Employees Prop. (Tangible-Internal) & $0.204 * * *$ & $0.0386^{* * *}$ & $0.0532 * * *$ \\
GS4. Beyond Compliance (Intangible-Internal) & $0.149 * * *$ & $0.148^{* * *}$ & 1 \\
\hline
\end{tabular}

$30.3 \%$ supply to public bodies. Thus, firms in the sample supply to more than one market segment.

Age, size and sector Following a large body of literature that suggests that age, size and industrial sector have an impact on firm growth (Coad 2008), we control for these effects. The average age of firms in the database is 24.20 years. The standard deviation of the age variable is 23.59 suggesting that there are several young as well as mature firms in the database. While $42.5 \%$ of the firms have less than 10 employees, $32.4 \%$ of the firms have more than equal to 10 and less than 50 employees, $18.1 \%$ of the firms have more than equal to 50 and less than 250 employees, and $6.95 \%$ of firms have more than 250 employees. The four main industry sectors of manufacturing, retail, services, and mining with other related industries are almost equally represented in the database.

Table 2 presents the correlations between the four greening variables. Although the correlations are significant suggesting that firms are engaged in multiple greening strategies, the correlations are small in magnitude. Table 3 summarises the adoption of the greening strategies across the age and size distribution of firms. In particular, the table suggests that the proportion of firms engaging in greening strategies increases along the age distribution and size distribution of firms. As the first row suggests, while $28.30 \%$ of firms that are less than or equal 10 years old have introduced a green product or service (GS1), 37.2\% of firms that are older than 50 years have introduced a green product or service. Similarly, while $28.8 \%$ of firms that have less than 50 employees have introduced a green product or service (GS1), 39.3\% of firms that have more than 250 employees have introduced a green product or service. This pattern exists for all the greening strategies.

\subsection{Estimation models}

For estimating the impact of the different types of greening processes on firm performance, ordered probit models are estimated in Tables 4, 5, 6, and 7, as the dependent variable is in an ordered form with firms experiencing a decrease in turnover, having the same turnover as in the previous period, or having an increased turnover. The core estimated equation is given as

$$
\begin{aligned}
y= & \alpha+\beta_{1}(G S 1)+\beta_{2}(G S 2)+\beta_{3}(G S 3) \\
& +\beta_{4}(G S 4)+\beta_{5}(\text { numgreen }) \\
& +\beta_{6}(\text { externalsupport })+\beta_{7}(\text { markettype }) \\
& +\beta_{8}(\text { firmage })+\beta_{9}(\text { firmsize })+\beta_{10}(\text { sector }) \\
& +\beta_{11}(\text { location })+e
\end{aligned}
$$

Table 3 Descriptive statistics

\begin{tabular}{llllllll}
\hline Greening Variable & All & $\begin{array}{l}\text { Age } \\
<=10 \text { years }\end{array}$ & $\begin{array}{l}\text { Age } \\
10-49 \text { years }\end{array}$ & $\begin{array}{l}\text { Age } \\
>=50 \text { years }\end{array}$ & $\begin{array}{l}\text { Size } \\
<50 \text { Emp. }\end{array}$ & $\begin{array}{l}\text { Size } \\
50-249 \text { Emp. }\end{array}$ & $\begin{array}{l}\text { Size } \\
>250 \text { Emp. }\end{array}$ \\
\hline GS1 & $30.30 \%$ & $28.30 \%$ & $30.10 \%$ & $37.20 \%$ & $28.80 \%$ & $33.30 \%$ & $39.30 \%$ \\
GS2 & $38.60 \%$ & $32.60 \%$ & $39.50 \%$ & $50.30 \%$ & $31.60 \%$ & $54.90 \%$ & $70.40 \%$ \\
GS3 & $43.80 \%$ & $40.70 \%$ & $43.70 \%$ & $53.30 \%$ & $39.00 \%$ & $53.80 \%$ & $68.70 \%$ \\
GS4 & $26.10 \%$ & $22.80 \%$ & $25.70 \%$ & $37.80 \%$ & $21.50 \%$ & $36.20 \%$ & $49.70 \%$ \\
N & 9236 & 2759 & 5505 & 972 & 6918 & 1676 & 642 \\
\hline
\end{tabular}


Table 4 Greening and firm performance

\begin{tabular}{|c|c|c|c|c|c|c|}
\hline & (1) & (2) & (3) & (4) & $(5)$ & $(6)$ \\
\hline Green Product or Service (Tangible-External) & $\begin{array}{l}0.164 * * * \\
(0.0413)\end{array}$ & & & & $\begin{array}{l}0.117 * * * \\
(0.0425)\end{array}$ & $\begin{array}{l}0.198 * * \\
(0.0775)\end{array}$ \\
\hline Env. Mang. Sys (Intangible-External) & & $\begin{array}{l}-0.0103 \\
(0.0415)\end{array}$ & & & $\begin{array}{l}-0.0199 \\
(0.0418)\end{array}$ & $\begin{array}{l}0.0436 \\
(0.0659)\end{array}$ \\
\hline Green Employees Prop. (Tangible-Internal) & & & $\begin{array}{l}0.132 * * * \\
(0.0437)\end{array}$ & & $\begin{array}{l}0.0949 * * * \\
(0.0363)\end{array}$ & $\begin{array}{l}0.114 * * * \\
(0.0429)\end{array}$ \\
\hline Beyond Compliance (Intangible-Internal) & & & & $\begin{array}{l}0.189 * * * \\
(0.0452)\end{array}$ & $\begin{array}{l}0.148 * * * \\
(0.0453)\end{array}$ & $\begin{array}{l}0.211 * * * \\
(0.0668)\end{array}$ \\
\hline Number of Green Strategies & & & & & & $\begin{array}{l}-0.0609 \\
(0.0476)\end{array}$ \\
\hline Financial Support & & & & & $\begin{array}{l}0.106 \\
(0.0651)\end{array}$ & $\begin{array}{l}0.115^{*} \\
(0.0653)\end{array}$ \\
\hline Non-financial Support & & & & & $\begin{array}{l}-0.0399 \\
(0.0408)\end{array}$ & $\begin{array}{l}-0.0345 \\
(0.0411)\end{array}$ \\
\hline Companies & & & & & $\begin{array}{l}0.242 * * * \\
(0.0397)\end{array}$ & $\begin{array}{l}0.243 * * * \\
(0.0397)\end{array}$ \\
\hline Public Bodies & & & & & $\begin{array}{l}0.0183 \\
(0.0426)\end{array}$ & $\begin{array}{l}0.0194 \\
(0.0425)\end{array}$ \\
\hline Age & $\begin{array}{l}-0.00976^{* * *} \\
(0.00113)\end{array}$ & $\begin{array}{l}-0.00966^{* * *} \\
(0.00113)\end{array}$ & $\begin{array}{l}-0.00973 * * * \\
(0.00114)\end{array}$ & $\begin{array}{l}-0.00956 * * * \\
(0.00114)\end{array}$ & $\begin{array}{l}-0.00945 * * * \\
(0.00115)\end{array}$ & $\begin{array}{l}-0.00946^{* * *} \\
(0.00115)\end{array}$ \\
\hline Employees: $10-49$ & $\begin{array}{l}0.433 * * * \\
(0.0444)\end{array}$ & $\begin{array}{l}0.442 * * * \\
(0.0445)\end{array}$ & $\begin{array}{l}0.454 * * * \\
(0.0445)\end{array}$ & $\begin{array}{l}0.432 * * * \\
(0.0444)\end{array}$ & $\begin{array}{l}0.420 * * * \\
(0.0450)\end{array}$ & $\begin{array}{l}0.424 * * * \\
(0.0451)\end{array}$ \\
\hline Employees: 50-249 & $\begin{array}{l}0.638 * * * \\
(0.0726)\end{array}$ & $\begin{array}{l}0.645 * * * \\
(0.0729)\end{array}$ & $\begin{array}{l}0.661 * * * \\
(0.0729)\end{array}$ & $\begin{array}{l}0.615 * * * \\
(0.0738)\end{array}$ & $\begin{array}{l}0.610 * * * \\
(0.0752)\end{array}$ & $\begin{array}{l}0.616^{* * *} \\
(0.0750)\end{array}$ \\
\hline Employees $>250$ & $\begin{array}{l}0.986 * * * \\
(0.141)\end{array}$ & $\begin{array}{l}1.011 * * * \\
(0.142)\end{array}$ & $\begin{array}{l}1.022 * * * \\
(0.140)\end{array}$ & $\begin{array}{l}0.947 * * * \\
(0.138)\end{array}$ & $\begin{array}{l}0.924 * * * \\
(0.142)\end{array}$ & $\begin{array}{l}0.941 * * * \\
(0.142)\end{array}$ \\
\hline Manufacturing & $\begin{array}{l}-0.0200 \\
(0.0529)\end{array}$ & $\begin{array}{l}-0.0269 \\
(0.0528)\end{array}$ & $\begin{array}{l}-0.0265 \\
(0.0528)\end{array}$ & $\begin{array}{l}-0.0217 \\
(0.0529)\end{array}$ & $\begin{array}{l}-0.0323 \\
(0.0539)\end{array}$ & $\begin{array}{l}-0.0327 \\
(0.0539)\end{array}$ \\
\hline Retail & $\begin{array}{l}0.0349 \\
(0.0491)\end{array}$ & $\begin{array}{l}0.0363 \\
(0.0490)\end{array}$ & $\begin{array}{l}0.0398 \\
(0.0490)\end{array}$ & $\begin{array}{l}0.0357 \\
(0.0490)\end{array}$ & $\begin{array}{l}0.0620 \\
(0.0493)\end{array}$ & $\begin{array}{l}0.0599 \\
(0.0494)\end{array}$ \\
\hline Services & $\begin{array}{l}0.00954 \\
(0.0478)\end{array}$ & $\begin{array}{l}-0.00707 \\
(0.0477)\end{array}$ & $\begin{array}{l}-0.00466 \\
(0.0477)\end{array}$ & $\begin{array}{l}-0.00824 \\
(0.0477)\end{array}$ & $\begin{array}{l}0.00525 \\
(0.0484)\end{array}$ & $\begin{array}{l}0.00331 \\
(0.0484)\end{array}$ \\
\hline Country Effects & Yes & Yes & Yes & Yes & Yes & Yes \\
\hline Constant cut 1 & $\begin{array}{l}-0.429 * * * \\
(0.150)\end{array}$ & $\begin{array}{l}-0.481 * * * \\
(0.150)\end{array}$ & $\begin{array}{l}-0.454 * * * \\
(0.150)\end{array}$ & $\begin{array}{l}-0.434 * * * \\
(0.148)\end{array}$ & $\begin{array}{l}-0.301 * * \\
(0.150)\end{array}$ & $\begin{array}{l}-0.311^{* *} \\
(0.150)\end{array}$ \\
\hline Constant cut 2 & $\begin{array}{l}0.389 * * * \\
(0.150)\end{array}$ & $\begin{array}{l}0.334 * * \\
(0.150)\end{array}$ & $\begin{array}{l}0.363 * * \\
(0.150)\end{array}$ & $\begin{array}{l}0.383 * * * \\
(0.148)\end{array}$ & $\begin{array}{l}0.526^{* * *} \\
(0.149)\end{array}$ & $\begin{array}{l}0.516^{* * * *} \\
(0.149)\end{array}$ \\
\hline Observations & 9236 & 9236 & 9236 & 9236 & 9236 & 9236 \\
\hline $\mathrm{R}^{2}$ & 0.0807 & 0.0790 & 0.0804 & 0.0809 & 0.0879 & 0.0881 \\
\hline$x^{2}$ & 728.5 & 707.6 & 725.2 & 726.9 & 814.1 & 819.5 \\
\hline
\end{tabular}

Robust standard errors in parentheses

$* * * p<0.01 ; * * p<0.05 ; * p<0.1$ 
Table 5 Greening and firm performance by firm age

\begin{tabular}{|c|c|c|c|}
\hline & $\begin{array}{l}(1) \\
\text { Age } \\
<=10 \text { years }\end{array}$ & $\begin{array}{l}(2) \\
\text { Age } \\
10-49 \text { years }\end{array}$ & $\begin{array}{l}(3) \\
\text { Age } \\
>50 \text { years }\end{array}$ \\
\hline Green Product or Service (Tangible-External) & $\begin{array}{l}0.448 * * * \\
(0.139)\end{array}$ & $\begin{array}{l}0.0876 \\
(0.102)\end{array}$ & $\begin{array}{l}0.344 \\
(0.257)\end{array}$ \\
\hline Env. Mang. Sys (Intangible-External) & $\begin{array}{l}0.139 \\
(0.119)\end{array}$ & $\begin{array}{l}0.0558 \\
(0.0854)\end{array}$ & $\begin{array}{l}0.210 \\
(0.254)\end{array}$ \\
\hline Green Employees Prop. (Tangible-Internal) & $\begin{array}{l}0.346^{* * * *} \\
(0.126)\end{array}$ & $\begin{array}{l}0.111 * * * \\
(0.0427)\end{array}$ & $\begin{array}{l}0.158 \\
(0.280)\end{array}$ \\
\hline Beyond Compliance (Intangible-Internal) & $\begin{array}{l}0.424 * * * \\
(0.123)\end{array}$ & $\begin{array}{l}0.151 * \\
(0.0867)\end{array}$ & $\begin{array}{l}0.133 \\
(0.278)\end{array}$ \\
\hline Number of Green Strategies & $\begin{array}{l}-0.189 * * \\
(0.0920)\end{array}$ & $\begin{array}{l}-0.0460 \\
(0.0612)\end{array}$ & $\begin{array}{l}-0.149 \\
(0.180)\end{array}$ \\
\hline Financial Support & $\begin{array}{l}0.124 \\
(0.103)\end{array}$ & $\begin{array}{l}0.109 \\
(0.0917)\end{array}$ & $\begin{array}{l}-0.106 \\
(0.285)\end{array}$ \\
\hline Non-financial Support & $\begin{array}{l}-0.0387 \\
(0.0691)\end{array}$ & $\begin{array}{l}-0.0496 \\
(0.0545)\end{array}$ & $\begin{array}{l}0.163 \\
(0.152)\end{array}$ \\
\hline Companies & $\begin{array}{l}0.302 * * * \\
(0.0640)\end{array}$ & $\begin{array}{l}0.210 * * * \\
(0.0538)\end{array}$ & $\begin{array}{l}0.186 \\
(0.162)\end{array}$ \\
\hline Public Bodies & $\begin{array}{l}0.115 \\
(0.0749)\end{array}$ & $\begin{array}{l}-0.0133 \\
(0.0553)\end{array}$ & $\begin{array}{l}0.247 \\
(0.154)\end{array}$ \\
\hline Employees: $10-49$ & $\begin{array}{l}0.634 * * * \\
(0.0807)\end{array}$ & $\begin{array}{l}0.434 * * * \\
(0.0584)\end{array}$ & $\begin{array}{l}0.314 \\
(0.201)\end{array}$ \\
\hline Employees: 50-249 & $\begin{array}{l}0.475^{* *} \\
(0.197)\end{array}$ & $\begin{array}{l}0.734 * * * \\
(0.0864)\end{array}$ & $\begin{array}{l}0.511 * * \\
(0.216)\end{array}$ \\
\hline Employees $>250$ & $\begin{array}{l}1.023 * * * \\
(0.370)\end{array}$ & $\begin{array}{l}0.906 * * * \\
(0.185)\end{array}$ & $\begin{array}{l}0.827 * * * \\
(0.274)\end{array}$ \\
\hline Age & $\begin{array}{l}-0.0584 * * * \\
(0.0112)\end{array}$ & $\begin{array}{l}-0.00827 * * * \\
(0.00286)\end{array}$ & $\begin{array}{l}0.00574 * \\
(0.00302)\end{array}$ \\
\hline Manufacturing & $\begin{array}{l}-0.0767 \\
(0.0927)\end{array}$ & $\begin{array}{l}0.0684 \\
(0.0728)\end{array}$ & $\begin{array}{l}-0.255 \\
(0.197)\end{array}$ \\
\hline Retail & $\begin{array}{l}0.0926 \\
(0.0814)\end{array}$ & $\begin{array}{l}0.0830 \\
(0.0664)\end{array}$ & $\begin{array}{l}0.0922 \\
(0.242)\end{array}$ \\
\hline Services & $\begin{array}{l}-0.0510 \\
(0.0757)\end{array}$ & $\begin{array}{l}0.0579 \\
(0.0682)\end{array}$ & $\begin{array}{l}-0.148 \\
(0.212)\end{array}$ \\
\hline Country Effects & Yes & Yes & Yes \\
\hline Constant cut 1 & $\begin{array}{l}-0.538^{* *} \\
(0.246)\end{array}$ & $\begin{array}{l}-0.247 \\
(0.222)\end{array}$ & $\begin{array}{l}0.409 \\
(0.359)\end{array}$ \\
\hline Constant cut 2 & $\begin{array}{l}0.295 \\
(0.245)\end{array}$ & $\begin{array}{l}0.634 * * * \\
(0.222)\end{array}$ & $\begin{array}{l}1.247 * * * \\
(0.365)\end{array}$ \\
\hline Observations & 2759 & 5505 & 972 \\
\hline $\mathrm{R}^{2}$ & 0.107 & 0.0923 & 0.138 \\
\hline$x^{2}$ & 379.5 & 517.9 & 185.8 \\
\hline
\end{tabular}

Robust standard errors in parentheses

$* * * p<0.01 ; * * p<0.05 ; * p<0.1$ 
Table 6 Greening and firm performance by firm size

\begin{tabular}{|c|c|c|c|}
\hline & $\begin{array}{l}(1) \\
\text { Employees } \\
<50\end{array}$ & $\begin{array}{l}(2) \\
\text { Employees } \\
50-249\end{array}$ & $\begin{array}{l}(3) \\
\text { Employees } \\
>250\end{array}$ \\
\hline \multirow[t]{2}{*}{ Green Product or Service (Tangible-External) } & $0.171 * *$ & $0.452 * *$ & 0.227 \\
\hline & $(0.0813)$ & $(0.198)$ & $(0.216)$ \\
\hline \multirow[t]{2}{*}{ Env. Mang. Sys (Intangible-External) } & 0.0321 & 0.216 & -0.162 \\
\hline & $(0.0686)$ & $(0.189)$ & $(0.262)$ \\
\hline \multirow[t]{2}{*}{ Green Employees Prop. (Tangible-Internal) } & $0.0734^{*}$ & 0.304 & $0.770 * *$ \\
\hline & $(0.0374)$ & $(0.237)$ & $(0.323)$ \\
\hline \multirow[t]{2}{*}{ Beyond Compliance (Intangible-Internal) } & $0.184 * * *$ & 0.255 & $0.764 * * *$ \\
\hline & $(0.0701)$ & $(0.181)$ & $(0.230)$ \\
\hline \multirow[t]{2}{*}{ Number of Green Strategies } & -0.0269 & -0.189 & -0.148 \\
\hline & $(0.0493)$ & $(0.134)$ & $(0.160)$ \\
\hline \multirow[t]{2}{*}{ Financial Support } & $0.172 * *$ & $-0.499 * * *$ & -0.129 \\
\hline & $(0.0697)$ & $(0.171)$ & $(0.244)$ \\
\hline \multirow[t]{2}{*}{ Non-financial Support } & 0.00208 & $-0.331 * * *$ & 0.0989 \\
\hline & $(0.0428)$ & $(0.121)$ & $(0.194)$ \\
\hline \multirow[t]{2}{*}{ Companies } & $0.272 * * *$ & 0.0741 & 0.263 \\
\hline & $(0.0412)$ & $(0.127)$ & $(0.189)$ \\
\hline \multirow[t]{2}{*}{ Public Bodies } & 0.0257 & 0.0400 & -0.114 \\
\hline & $(0.0447)$ & $(0.115)$ & $(0.180)$ \\
\hline \multirow[t]{2}{*}{ Age } & $-0.00938 * * *$ & 0.000671 & 0.000322 \\
\hline & $(0.00128)$ & $(0.00216)$ & $(0.00244)$ \\
\hline \multirow[t]{2}{*}{ Manufacturing } & -0.0360 & $0.326 * *$ & 0.131 \\
\hline & $(0.0577)$ & $(0.134)$ & $(0.224)$ \\
\hline \multirow[t]{2}{*}{ Retail } & 0.0482 & $0.445 * * *$ & $-0.576^{*}$ \\
\hline & $(0.0510)$ & $(0.164)$ & $(0.316)$ \\
\hline \multirow[t]{2}{*}{ Services } & 0.0135 & $0.338 * *$ & $0.639 * * *$ \\
\hline & $(0.0502)$ & $(0.152)$ & $(0.240)$ \\
\hline Country Effects & Yes & Yes & Yes \\
\hline \multirow[t]{2}{*}{ Constant cut 1} & $-0.363 * *$ & -0.139 & $-0.959^{*}$ \\
\hline & $(0.154)$ & $(0.524)$ & $(0.546)$ \\
\hline \multirow[t]{2}{*}{ Constant cut 2} & $0.463 * * *$ & 0.678 & -0.109 \\
\hline & $(0.154)$ & $(0.516)$ & $(0.558)$ \\
\hline Observations & 6918 & 1676 & 642 \\
\hline $\mathrm{R}^{2}$ & 0.0800 & 0.107 & 0.270 \\
\hline$x^{2}$ & 657.3 & 175.6 & 1286 \\
\hline
\end{tabular}

Robust standard errors in parentheses

$* * * p<0.01 ; * * p<0.05 ; * p<0.1$

where GS1-GS4 are the four greening strategies (tangible-external, intangible-external, tangibleinternal and intangible-internal). In addition to these core independent variables and firm age and size controls, numgreen controls for the number of green strategies adopted a firm, externalsupport for financial and non-financial external support received by a firm, markettype for the different types of markets a 
Table 7 Greening and firm performance (multiple greening strategies)

\begin{tabular}{|c|c|c|c|c|}
\hline & (1) & (2) & (3) & (4) \\
\hline Green Product or Service (Tangible-External) & & $\begin{array}{l}0.198 * * \\
(0.0775)\end{array}$ & & $\begin{array}{l}0.186^{* *} \\
(0.0781)\end{array}$ \\
\hline Env. Mang. Sys (Intangible-External) & & $\begin{array}{l}0.0436 \\
(0.0659)\end{array}$ & & $\begin{array}{l}0.0357 \\
(0.0669)\end{array}$ \\
\hline Green Employees Prop. (Tangible-Internal) & & $\begin{array}{l}0.114 * * * \\
(0.0429)\end{array}$ & & $\begin{array}{l}0.112 * * * \\
(0.0424)\end{array}$ \\
\hline Beyond Compliance (Intangible-Internal) & & $\begin{array}{l}0.211 * * * \\
(0.0668)\end{array}$ & & $\begin{array}{l}0.196 * * * \\
(0.0684)\end{array}$ \\
\hline Number of Green Strategies & $\begin{array}{l}0.0654 * * * \\
(0.0175)\end{array}$ & $\begin{array}{l}-0.0609 \\
(0.0476)\end{array}$ & & \\
\hline 1 Green Strategy & & & $\begin{array}{l}0.0127 \\
(0.0445)\end{array}$ & $\begin{array}{l}-0.0871 \\
(0.0636)\end{array}$ \\
\hline 2 Green Strategies & & & $\begin{array}{l}0.0642 \\
(0.0511)\end{array}$ & $\begin{array}{l}-0.154 \\
(0.0982)\end{array}$ \\
\hline 3 Green Strategies & & & $\begin{array}{l}0.213 * * * \\
(0.0671)\end{array}$ & $\begin{array}{l}-0.146 \\
(0.151)\end{array}$ \\
\hline 4 Green Strategies & & & $\begin{array}{l}0.354 * * * \\
(0.117)\end{array}$ & $\begin{array}{l}-0.129 \\
(0.224)\end{array}$ \\
\hline Financial Support & $\begin{array}{l}0.109^{*} \\
(0.0651)\end{array}$ & $\begin{array}{l}0.115^{*} \\
(0.0653)\end{array}$ & $\begin{array}{l}0.110^{*} \\
(0.0653)\end{array}$ & $\begin{array}{l}0.115^{*} \\
(0.0655)\end{array}$ \\
\hline Non-financial Support & $\begin{array}{l}-0.0320 \\
(0.0408)\end{array}$ & $\begin{array}{l}-0.0345 \\
(0.0411)\end{array}$ & $\begin{array}{l}-0.0342 \\
(0.0407)\end{array}$ & $\begin{array}{l}-0.0360 \\
(0.0410)\end{array}$ \\
\hline Companies & $\begin{array}{l}0.246^{* * * *} \\
(0.0398)\end{array}$ & $\begin{array}{l}0.243 * * * \\
(0.0397)\end{array}$ & $\begin{array}{l}0.245 * * * \\
(0.0397)\end{array}$ & $\begin{array}{l}0.242 * * * \\
(0.0397)\end{array}$ \\
\hline Public Bodies & $\begin{array}{l}0.0244 \\
(0.0425)\end{array}$ & $\begin{array}{l}0.0194 \\
(0.0425)\end{array}$ & $\begin{array}{l}0.0246 \\
(0.0425)\end{array}$ & $\begin{array}{l}0.0200 \\
(0.0426)\end{array}$ \\
\hline Age & $\begin{array}{l}-0.00949 * * * \\
(0.00115)\end{array}$ & $\begin{array}{l}-0.00946^{* * * *} \\
(0.00115)\end{array}$ & $\begin{array}{l}-0.00948 * * * \\
(0.00115)\end{array}$ & $\begin{array}{l}-0.00946^{* * *} \\
(0.00116)\end{array}$ \\
\hline Employees: $10-49$ & $\begin{array}{l}0.408^{* * * *} \\
(0.0448)\end{array}$ & $\begin{array}{l}0.424 * * * \\
(0.0451)\end{array}$ & $\begin{array}{l}0.406^{* * * *} \\
(0.0448)\end{array}$ & $\begin{array}{l}0.422 * * * \\
(0.0451)\end{array}$ \\
\hline Employees: 50-249 & $\begin{array}{l}0.581 * * * \\
(0.0752)\end{array}$ & $\begin{array}{l}0.616 * * * \\
(0.0750)\end{array}$ & $\begin{array}{l}0.573 * * * \\
(0.0756)\end{array}$ & $\begin{array}{l}0.609 * * * \\
(0.0754)\end{array}$ \\
\hline Employees $>250$ & $\begin{array}{l}0.890 * * * \\
(0.141)\end{array}$ & $\begin{array}{l}0.941 * * * \\
(0.142)\end{array}$ & $\begin{array}{l}0.870 * * * \\
(0.141)\end{array}$ & $\begin{array}{l}0.925 * * * \\
(0.142)\end{array}$ \\
\hline Manufacturing & $\begin{array}{l}-0.0337 \\
(0.0538)\end{array}$ & $\begin{array}{l}-0.0327 \\
(0.0539)\end{array}$ & $\begin{array}{l}-0.0325 \\
(0.0539)\end{array}$ & $\begin{array}{l}-0.0318 \\
(0.0540)\end{array}$ \\
\hline Retail & $\begin{array}{l}0.0683 \\
(0.0493)\end{array}$ & $\begin{array}{l}0.0599 \\
(0.0494)\end{array}$ & $\begin{array}{l}0.0682 \\
(0.0493)\end{array}$ & $\begin{array}{l}0.0604 \\
(0.0494)\end{array}$ \\
\hline Services & $\begin{array}{l}0.00888 \\
(0.0484)\end{array}$ & $\begin{array}{l}0.00331 \\
(0.0484)\end{array}$ & $\begin{array}{l}0.00912 \\
(0.0484)\end{array}$ & $\begin{array}{l}0.00405 \\
(0.0484)\end{array}$ \\
\hline Country Effects & Yes & Yes & Yes & Yes \\
\hline Constant cut 1 & $\begin{array}{l}-0.317 * * \\
(0.152)\end{array}$ & $\begin{array}{l}-0.311 * * \\
(0.150)\end{array}$ & $\begin{array}{l}-0.349 * * \\
(0.152)\end{array}$ & $\begin{array}{l}-0.334 * * \\
(0.150)\end{array}$ \\
\hline Constant cut 2 & $\begin{array}{l}0.507 * * * \\
(0.152)\end{array}$ & $\begin{array}{l}0.516^{* * * *} \\
(0.149)\end{array}$ & $\begin{array}{l}0.475 * * * \\
(0.152)\end{array}$ & $\begin{array}{l}0.493 * * * \\
(0.150)\end{array}$ \\
\hline Observations & 9236 & 9236 & 9236 & 9236 \\
\hline $\mathrm{R}^{2}$ & 0.0858 & 0.0881 & 0.0863 & 0.0883 \\
\hline$x^{2}$ & 787.5 & 819.5 & 793.1 & 823.3 \\
\hline
\end{tabular}

Robust standard errors in parentheses

$* * * p<0.01 ; * * p<0.05 ; * p<0.1$ 
firm caters to, sector for the industrial sector and location for the country of a firm's geographic location.

\section{Empirical results}

The empirical results linking the four greening strategies with firm performance are presented in Table 4. As the positive and significant coefficient of the 'Green Product or Service' variable in column (1) suggests, tangibleexternal greening strategy has a positive impact on firm performance. However, the coefficient of 'Env. Mang. Sys.' in column (2) is insignificant suggesting that an intangible-external greening strategy has no impact on firm performance. The coefficients of the internal greening variables in columns (3) and (4) suggest that both forms of internal greening have a positive impact on firm performance. In column (5), all greening variables are jointly introduced in the estimation, and in column (6) the number of greening strategies pursued by the firms is introduced in the estimation as an additional control. The results in column (6) are consistent with the results in columns (2), (3), (4), and (5). Thus, the results partially support hypothesis H1a while strongly supporting hypothesis $\mathrm{H}_{1} \mathrm{~b}{ }^{6}$

The estimated effects of the control variables suggest that while external financial support and offering products and services to companies have significantly positive effects, the number of greening strategies and nonfinancial support have an insignificant effect on firm performance.

While the empirical results in Table 4 suggest that greening strategies have a positive impact on firm

\footnotetext{
${ }^{6}$ The marginal effects of the ordered probit estimation in column (6) of Table 4 are presented in Table 8 to quantify the estimated impact of greening strategies on firm performance. The estimated marginal effects suggest that while introducing a green product or service increases the likelihood of a firm reporting increased turnover by 7.4 percentage points, a $1 \%$ increase in the proportion of green employees increases the likelihood of a firm reporting increased turnover by 4.2 percentage points, and going beyond compliance requirements increases the likelihood of a firm reporting increased turnover by 7.9 percentage points. For brevity, we have not included the marginal effect estimations for other regressions equations in the paper, but these are available from the authors. Furthermore, we check for potential biases resulting from data collection and the robustness of results presented in Table 3 by excluding firms following all four greening strategies from the sample in Table 9. The empirical results are consistent with the results in Table 3, and confirm the robustness of the results presented in Table 3 .
}

performance, they do not show which type of greening matters for whom. In Table 5, we examine the impact for greening on firm performance along the age distribution to test the hypotheses $\mathrm{H} 2$ and $\mathrm{H} 3$ in the context of young, middle-aged and old firms. Consistent with the hypothesis $\mathrm{H} 2 \mathrm{a}$, the results in column (1) suggest that having a tangible-external greening strategy in the form of offering a green product or service has resulted in an increase in turnover for young entrepreneurial firms. However, consistent with hypothesis $\mathrm{H} 2 \mathrm{~b}$, the coefficients for the 'Tangible-External' variable in columns (2) and (3) are insignificant suggesting that tangible-external greening has no impact on middle-aged and mature firms. Consistent with the hypothesis H3a, the internal greening strategies have a positive impact on firm performance for young firms as suggested by the positive coefficients on 'Green Employees Prop.' and 'Beyond Compliance' variables in column (1). Although these coefficients are positive and significant for middle-aged firms, they are insignificant for the oldest firms suggesting that internal greening strategies have a positive impact on the performance for young and middle-aged firms but not old firms. Thus, the results partially support H3b.

In Table 6, the impact of greening strategies on firm performance is examined along the sized distribution of the firms in the database to test the hypotheses $\mathrm{H} 2$ and $\mathrm{H} 3$ in the context of small, mid-sized, and large firms. Consistent with the hypothesis $\mathrm{H} 2 \mathrm{a}$, the results suggest that tangibleexternal greening strategy has a positive impact on firm performance for small firms, and consistent with the hypothesis $\mathrm{H} 2 \mathrm{~b}$, the impact of tangibleexternal greening strategy decreases along the size distribution. Although it benefits mid-sized firms, it has no significant impact on the performance of large firms. Consistent with the hypothesis H3a, both tangible as well as intangible internal greening strategies have a positive impact on the performance of small firms. While they do not have a significant impact on performance for mid-sized firms, the internal greening strategies have a positive impact on the performance of large firms suggesting that as firms mature, they need to move from external greening strategies to internal greening strategies. Thus, the results partially support $\mathrm{H} 3 \mathrm{~b}$. 
An important aspect of the above results is that they do not explicitly consider firms pursuing multiple greening strategies. To address this issue, in Table 7, the impact of following multiple greening strategies on firm performance is estimated in column (1). The positive and significant coefficient of the variable 'Num. Green Strategies' suggests that following multiple greening strategies has a positive impact on firm performance. However, cointroducing the four greening strategies in column (2) makes the coefficient of the variable 'Num. Green Strategies' insignificant suggesting that the primary effects of following these greening strategies are central to explaining the impact of greening on firm performance. These results are replicated in columns (3) and (4) that use dummy variables to indicate the number of greening strategies adopted by a firm. The results in column (3) suggest that simultaneously pursuing 3 or 4 greening strategies has a positive impact on firm performance. However, these effects disappear once the four greening strategies are introduced in column (4). Thus, the results in Table 7 suggest that the primary effects have a direct impact on firm performance. ${ }^{7}$

\section{Conclusions}

With ever-increasing consciousness about the environment, there is an increasing demand for sustainability-driven products and services. Emerging studies along these lines suggest that sustainable development can be used by firms as a means of earning profits (Cohen and Winn 2007; Dean and McMullen 2007). This has led to a position that sustainable development and green practices are routes to competitive advantage (Esty and Winston 2009; King and Lenox 2001). However, this broad literature uses several different forms of greening interchangeably to examine the impact of greening for competitive advantage and performance. The

\footnotetext{
${ }^{7}$ Table 10 presents a summary table showing the co-occurrence of multiple greening strategies. To further examine the role of multiplicity of greening strategies for firm performance and check for robustness of these results, Table 11 explicitly introduces multiple greening strategies variables in the estimation. The empirical results are broadly consistent with the results of Table 7 and suggest that the effects of the four greening strategies can explain the impact of greening on firm performance.
}

greening framework model developed in Section 2 provides a basis for underpinning the role of different types of greening initiatives for firm performance and shows which type of greening matters for whom. In particular, the results suggest that while both external and internal greening strategies have an impact on firm performance for young firms and small firms, internal greening strategies are more important for middle-aged firms and large firms.

Young entrepreneurial firms are more likely to target an unaddressed niche to increases their likelihood of survival in the face of competition from large incumbents (Bhide 1994). This potentially results in the same external greening strategy having a differential impact on firm performance across the age distribution. For these young firms, tangibleexternal greening provides an opportunity to deal with the liability of newness (Stinchcombe 1965). Although green product innovation literature addresses environmental issues explicitly, it is far from certain whether these products can truly achieve market success (Pujari 2006). In this context, the results suggest that green product innovation may be more crucial for entrepreneurial firms than incumbents.

Furthermore, the results suggest that as firms mature, they need to change their greening strategies and shift from external greening to internal greening activities. One potential cause may be that as firms mature, they need to undertake demonstrable internal greening activities to affirm their green commitments to employees and other stakeholders. In addition to this, the results suggest that although firms may follow multiple greening strategies, the effects of the primary four greening strategies can directly explain the variation in firm performance that is attributable to greening.

The main limitation of this study is that the measure of firm performance is a categorical variable that measures if a firm's turnover has decreased, remained same, or increased over the last 2 years while the magnitude of this change is not known. A change over a 2-year period can provide limited insight into the determinants of firm performance over a longer time period. This is particularly relevant as recent research suggests that there is inherent randomness in firm growth (Coad et al. 2013). In addition to this, most of the independent variables, 
with the exception of the age variable, are categorical in nature. Future research can overcome these limitations by using an alternate database that has continuous data over a longer time period. Furthermore, future research can examine the impact of these greening strategies across different industry sectors and country contexts, particularly with regard to the multiplicity of greening strategies.

To conclude, the paper presents a novel framework for contextualising greening by firms through the lens of tangibility and visibility of its greening activities. By classifying greening actives as externally tangible, externally intangible, internally tangible and internally intangible, the paper provides a compelling tool for examining how greening activities impact firm performance. Furthermore, by examining the impact of greening along the age distribution and the size distribution of firms, the paper provides novel insights into the role of age and size for the link between greening and performance.

\section{Appendix}

Table 8 Greening and firm performance (marginal effects of Table 4 col. (6))

\begin{tabular}{|c|c|c|c|}
\hline & $\begin{array}{l}\text { (1) } \\
\text { Decrease }\end{array}$ & $\begin{array}{l}\text { (2) } \\
\text { No Change }\end{array}$ & $\begin{array}{l}\text { (3) } \\
\text { Increase }\end{array}$ \\
\hline Green Product or Service (Tangible-External) & $\begin{array}{l}-0.071 * * * \\
(0.009)\end{array}$ & $\begin{array}{l}-0.002 \\
(0.281)\end{array}$ & $\begin{array}{l}0.074 * * \\
(0.012)\end{array}$ \\
\hline Env. Mang. Sys (Intangible-External) & $\begin{array}{l}-0.016 \\
(0.506)\end{array}$ & $\begin{array}{l}-0.000 \\
(0.823)\end{array}$ & $\begin{array}{l}0.016 \\
(0.509)\end{array}$ \\
\hline Green Employees Prop. (Tangible-Internal) & $\begin{array}{l}-0.042 * * * \\
(0.008)\end{array}$ & $\begin{array}{l}0.000 \\
(0.898)\end{array}$ & $\begin{array}{l}0.042 * * * \\
(0.008)\end{array}$ \\
\hline Beyond Compliance (Intangible-Internal) & $\begin{array}{l}-0.075 * * * \\
(0.001)\end{array}$ & $\begin{array}{l}-0.004 \\
(0.161)\end{array}$ & $\begin{array}{l}0.079 * * * \\
(0.002)\end{array}$ \\
\hline Number of Green Strategies & $\begin{array}{l}0.022 \\
(0.201)\end{array}$ & $\begin{array}{l}-0.000 \\
(0.898)\end{array}$ & $\begin{array}{l}-0.022 \\
(0.201)\end{array}$ \\
\hline Financial Support & $\begin{array}{l}-0.041 * \\
(0.072)\end{array}$ & $\begin{array}{l}-0.002 \\
(0.412)\end{array}$ & $\begin{array}{l}0.043 * \\
(0.083)\end{array}$ \\
\hline Non-financial Support & $\begin{array}{l}0.013 \\
(0.402)\end{array}$ & $\begin{array}{l}-0.000 \\
(0.739)\end{array}$ & $\begin{array}{l}-0.013 \\
(0.399)\end{array}$ \\
\hline Companies & $\begin{array}{l}-0.090 \text { *** } \\
(0.000)\end{array}$ & $\begin{array}{l}0.003 * \\
(0.091)\end{array}$ & $\begin{array}{l}0.088 * * * \\
(0.000)\end{array}$ \\
\hline Public Bodies & $\begin{array}{l}-0.007 \\
(0.648)\end{array}$ & $\begin{array}{l}-0.000 \\
(0.931)\end{array}$ & $\begin{array}{l}0.007 \\
(0.649)\end{array}$ \\
\hline Age & $\begin{array}{l}0.003 * * * \\
(0.000)\end{array}$ & $\begin{array}{l}-0.000 \\
(0.898)\end{array}$ & $\begin{array}{l}-0.003 * * * \\
(0.000)\end{array}$ \\
\hline Employees: 10-49 & $\begin{array}{l}-0.145^{* * *} \\
(0.000)\end{array}$ & $\begin{array}{l}-0.017 * * * \\
(0.000)\end{array}$ & $\begin{array}{l}0.162 * * * \\
(0.000)\end{array}$ \\
\hline Employees: 50-249 & $\begin{array}{l}-0.192 * * * \\
(0.000)\end{array}$ & $\begin{array}{l}-0.048^{* * * *} \\
(0.000)\end{array}$ & $\begin{array}{l}0.240 * * * \\
(0.000)\end{array}$ \\
\hline Employees $>250$ & $\begin{array}{l}-0.255^{* * *} \\
(0.000)\end{array}$ & $\begin{array}{l}-0.106^{* * *} \\
(0.000)\end{array}$ & $\begin{array}{l}0.362 * * * \\
(0.000)\end{array}$ \\
\hline Industry Effects & Yes & Yes & Yes \\
\hline Country Effects & Yes & Yes & Yes \\
\hline Observations & 9236 & & \\
\hline $\mathrm{R}^{2}$ & 0.0881 & & \\
\hline$\chi^{2}$ & 819.5 & & \\
\hline
\end{tabular}


Table 9 Greening and firm performance (robustness checks)

\begin{tabular}{|c|c|c|c|c|c|c|}
\hline & (1) & (2) & (3) & (4) & $(5)$ & $(6)$ \\
\hline Green Product or Service (Tangible-External) & $\begin{array}{l}0.144 * * * \\
(0.0430)\end{array}$ & & & & $\begin{array}{l}0.111 * * \\
(0.0437)\end{array}$ & $\begin{array}{l}0.195^{* *} \\
(0.0778)\end{array}$ \\
\hline Env. Mang. Sys (Intangible-External) & & $\begin{array}{l}-0.0408 \\
(0.0429)\end{array}$ & & & $\begin{array}{l}-0.0275 \\
(0.0431)\end{array}$ & $\begin{array}{l}0.0382 \\
(0.0662)\end{array}$ \\
\hline Green Employees Prop. (Tangible-Internal) & & & $\begin{array}{l}0.114 * * * \\
(0.0400)\end{array}$ & & $\begin{array}{l}0.0875 * * \\
(0.0358)\end{array}$ & $\begin{array}{l}0.108 * * * \\
(0.0415)\end{array}$ \\
\hline Beyond Compliance (Intangible-Internal) & & & & $\begin{array}{l}0.169 * * * \\
(0.0476)\end{array}$ & $\begin{array}{l}0.141 * * * \\
(0.0474)\end{array}$ & $\begin{array}{l}0.207 * * * \\
(0.0677)\end{array}$ \\
\hline Number of Green Strategies & & & & & & $\begin{array}{l}-0.0640 \\
(0.0476)\end{array}$ \\
\hline Financial Support & & & & & $\begin{array}{l}0.105 \\
(0.0663)\end{array}$ & $\begin{array}{l}0.114 * \\
(0.0665)\end{array}$ \\
\hline Non-financial Support & & & & & $\begin{array}{l}-0.0455 \\
(0.0415)\end{array}$ & $\begin{array}{l}-0.0397 \\
(0.0417)\end{array}$ \\
\hline Companies & & & & & $\begin{array}{l}0.239^{* * * *} \\
(0.0403)\end{array}$ & $\begin{array}{l}0.240 * * * \\
(0.0403)\end{array}$ \\
\hline Public Bodies & & & & & $\begin{array}{l}0.0228 \\
(0.0434)\end{array}$ & $\begin{array}{l}0.0241 \\
(0.0434)\end{array}$ \\
\hline Age & $\begin{array}{l}-0.00992 * * * \\
(0.00118)\end{array}$ & $\begin{array}{l}-0.00986^{* * * *} \\
(0.00118)\end{array}$ & $\begin{array}{l}-0.00993 * * * \\
(0.00119)\end{array}$ & $\begin{array}{l}-0.00972 * * * \\
(0.00119)\end{array}$ & $\begin{array}{l}-0.00960 * * * \\
(0.00120)\end{array}$ & $\begin{array}{l}-0.00961 * * * \\
(0.00120)\end{array}$ \\
\hline Employees: 10-49 & $\begin{array}{l}0.437 * * * \\
(0.0452)\end{array}$ & $\begin{array}{l}0.443 * * * \\
(0.0453)\end{array}$ & $\begin{array}{l}0.453 * * * \\
(0.0453)\end{array}$ & $\begin{array}{l}0.437 * * * \\
(0.0452)\end{array}$ & $\begin{array}{l}0.424 * * * \\
(0.0458)\end{array}$ & $\begin{array}{l}0.428 * * * \\
(0.0459)\end{array}$ \\
\hline Employees: 50-249 & $\begin{array}{l}0.664 * * * \\
(0.0760)\end{array}$ & $\begin{array}{l}0.668 * * * \\
(0.0763)\end{array}$ & $\begin{array}{l}0.677 * * * \\
(0.0765)\end{array}$ & $\begin{array}{l}0.644 * * * \\
(0.0770)\end{array}$ & $\begin{array}{l}0.645 * * * \\
(0.0782)\end{array}$ & $\begin{array}{l}0.651 * * * \\
(0.0780)\end{array}$ \\
\hline Employees $>250$ & $\begin{array}{l}0.919 * * * \\
(0.154)\end{array}$ & $\begin{array}{l}0.930 * * * \\
(0.154)\end{array}$ & $\begin{array}{l}0.933 * * * \\
(0.152)\end{array}$ & $\begin{array}{l}0.881^{* * *} \\
(0.150)\end{array}$ & $\begin{array}{l}0.869^{* * * *} \\
(0.153)\end{array}$ & $\begin{array}{l}0.887 * * * \\
(0.153)\end{array}$ \\
\hline Industry Effects & Yes & Yes & Yes & Yes & Yes & Yes \\
\hline Country Effects & Yes & Yes & Yes & Yes & Yes & Yes \\
\hline Constant cut 1 & $\begin{array}{l}-0.438 * * * \\
(0.151)\end{array}$ & $\begin{array}{l}-0.488 * * * \\
(0.151)\end{array}$ & $\begin{array}{l}-0.462^{* * *} \\
(0.151)\end{array}$ & $\begin{array}{l}-0.442 * * * \\
(0.150)\end{array}$ & $\begin{array}{l}-0.312^{* *} \\
(0.151)\end{array}$ & $\begin{array}{l}-0.323 * * \\
(0.151)\end{array}$ \\
\hline Constant cut 2 & $\begin{array}{l}0.388^{* *} \\
(0.151)\end{array}$ & $\begin{array}{l}0.337 * * \\
(0.151)\end{array}$ & $\begin{array}{l}0.363 * * \\
(0.151)\end{array}$ & $\begin{array}{l}0.384 * * \\
(0.149)\end{array}$ & $\begin{array}{l}0.523^{* * * *} \\
(0.151)\end{array}$ & $\begin{array}{l}0.512 * * * \\
(0.151)\end{array}$ \\
\hline Observations & 8757 & 8757 & 8757 & 8757 & 8757 & 8757 \\
\hline $\mathrm{R}^{2}$ & 0.0792 & 0.0780 & 0.0790 & 0.0793 & 0.0860 & 0.0862 \\
\hline$X^{2}$ & 690.2 & 675.3 & 691.8 & 688.4 & 768.8 & 775.6 \\
\hline
\end{tabular}

Robust standard errors in parentheses

$* * * p<0.01 ; * * p<0.05, * p<0.1$

The sample excludes firms that reported following all four greening strategies 
Table 10 Multiplicity of greening strategies

\begin{tabular}{ll}
\hline GS1 Green Product or Service (Tangible-External) & $30.30 \%$ \\
GS2 Env. Mang. Sys (Intangible-External) & $38.60 \%$ \\
GS3 Has Green Employees & $43.80 \%$ \\
GS4 Beyond Compliance (Intangible-Internal) & $26.10 \%$ \\
GS12 & $13.20 \%$ \\
GS13 & $22.00 \%$ \\
GS14 & $10.90 \%$ \\
GS23 & $21.00 \%$ \\
GS24 & $13.20 \%$ \\
GS34 & $14.90 \%$ \\
GS123 & $10.60 \%$ \\
GS124 & $6.12 \%$ \\
GS134 & $8.56 \%$ \\
GS234 & $9.00 \%$ \\
GS1234 & $5.19 \%$ \\
N & 9236 \\
\hline
\end{tabular}

Table 11 Greening and firm performance (multiplicity of greening strategies)

\begin{tabular}{|c|c|c|c|}
\hline & 2 Modes & 3 Modes & 4 Modes \\
\hline GS1. Green Product or Service (Tangible-External) & $\begin{array}{l}0.213 * * \\
(0.0889)\end{array}$ & $\begin{array}{l}0.251 * * * \\
(0.0905)\end{array}$ & $\begin{array}{l}0.252 * * * \\
(0.0906)\end{array}$ \\
\hline GS2. Env. Mang. Sys (Intangible-External) & $\begin{array}{l}0.0720 \\
(0.0737)\end{array}$ & $\begin{array}{l}0.0820 \\
(0.0756)\end{array}$ & $\begin{array}{l}0.0821 \\
(0.0756)\end{array}$ \\
\hline GS3. Green Employees Prop. (Tangible-Internal) & $\begin{array}{l}0.0877 * * \\
(0.0435)\end{array}$ & $\begin{array}{l}0.101 * * \\
(0.0435)\end{array}$ & $\begin{array}{l}0.101 * * \\
(0.0434)\end{array}$ \\
\hline GS4. Beyond Compliance (Intangible-Internal) & $\begin{array}{l}0.138 * \\
(0.0766)\end{array}$ & $\begin{array}{l}0.176^{* *} \\
(0.0803)\end{array}$ & $\begin{array}{l}0.175^{* *} \\
(0.0803)\end{array}$ \\
\hline GS12 & $\begin{array}{l}-0.0913 \\
(0.0905)\end{array}$ & $\begin{array}{l}-0.188^{*} \\
(0.110)\end{array}$ & $\begin{array}{l}-0.187 * \\
(0.111)\end{array}$ \\
\hline GS13 & $\begin{array}{l}-0.0170 \\
(0.0730)\end{array}$ & $\begin{array}{l}-0.108 \\
(0.0943)\end{array}$ & $\begin{array}{l}-0.106 \\
(0.0966)\end{array}$ \\
\hline GS14 & $\begin{array}{l}0.0916 \\
(0.0984)\end{array}$ & $\begin{array}{l}-0.0721 \\
(0.127)\end{array}$ & $\begin{array}{l}-0.0693 \\
(0.131)\end{array}$ \\
\hline GS23 & $\begin{array}{l}-0.000627 \\
(0.0716)\end{array}$ & $\begin{array}{l}0.0315 \\
(0.149)\end{array}$ & $\begin{array}{l}0.0369 \\
(0.162)\end{array}$ \\
\hline GS24 & $\begin{array}{l}0.0275 \\
(0.0968)\end{array}$ & $\begin{array}{l}-0.0505 \\
(0.124)\end{array}$ & $\begin{array}{l}-0.0477 \\
(0.127)\end{array}$ \\
\hline GS34 & $\begin{array}{l}0.203 * * \\
(0.0943)\end{array}$ & $\begin{array}{l}0.0761 \\
(0.207)\end{array}$ & $\begin{array}{l}0.0861 \\
(0.255)\end{array}$ \\
\hline GS123 & & $\begin{array}{l}0.0527 \\
(0.170)\end{array}$ & $\begin{array}{l}0.0444 \\
(0.191)\end{array}$ \\
\hline GS124 & & $\begin{array}{l}0.304 \\
(0.206)\end{array}$ & $\begin{array}{l}0.296 \\
(0.227)\end{array}$ \\
\hline GS134 & & $\begin{array}{l}0.302 \\
(0.219)\end{array}$ & $\begin{array}{l}0.286 \\
(0.306)\end{array}$ \\
\hline GS234 & & $\begin{array}{l}-0.156 \\
(0.212)\end{array}$ & $\begin{array}{l}-0.180 \\
(0.338)\end{array}$ \\
\hline
\end{tabular}


Table 11 (continued)

\begin{tabular}{|c|c|c|c|}
\hline & 2 Modes & 3 Modes & 4 Modes \\
\hline GS1234 & & & $\begin{array}{l}0.0391 \\
(0.441)\end{array}$ \\
\hline Number of Green Strategies & $\begin{array}{l}-0.0692 \\
(0.0464)\end{array}$ & $\begin{array}{l}-0.0641 \\
(0.0471)\end{array}$ & $\begin{array}{l}-0.0647 \\
(0.0478)\end{array}$ \\
\hline Financial Support & $\begin{array}{l}0.118^{*} \\
(0.0654)\end{array}$ & $\begin{array}{l}0.115^{*} \\
(0.0655)\end{array}$ & $\begin{array}{l}0.115^{*} \\
(0.0655)\end{array}$ \\
\hline Non-financial Support & $\begin{array}{l}-0.0345 \\
(0.0411)\end{array}$ & $\begin{array}{l}-0.0324 \\
(0.0410)\end{array}$ & $\begin{array}{l}-0.0324 \\
(0.0410)\end{array}$ \\
\hline Companies & $\begin{array}{l}0.242 * * * \\
(0.0397)\end{array}$ & $\begin{array}{l}0.241 * * * \\
(0.0398)\end{array}$ & $\begin{array}{l}0.241 * * * \\
(0.0398)\end{array}$ \\
\hline Public Bodies & $\begin{array}{l}0.0216 \\
(0.0426)\end{array}$ & $\begin{array}{l}0.0227 \\
(0.0426)\end{array}$ & $\begin{array}{l}0.0227 \\
(0.0426)\end{array}$ \\
\hline Age & $\begin{array}{l}-0.00950 * * * \\
(0.00115)\end{array}$ & $\begin{array}{l}-0.00958 * * * \\
(0.00115)\end{array}$ & $\begin{array}{l}-0.00958^{* * * *} \\
(0.00115)\end{array}$ \\
\hline Employees: 10-49 & $\begin{array}{l}0.426 * * * \\
(0.0451)\end{array}$ & $\begin{array}{l}0.425 * * * \\
(0.0452)\end{array}$ & $\begin{array}{l}0.425 * * * \\
(0.0452)\end{array}$ \\
\hline Employees: 50-249 & $\begin{array}{l}0.624 * * * \\
(0.0753)\end{array}$ & $\begin{array}{l}0.624 * * * \\
(0.0755)\end{array}$ & $\begin{array}{l}0.624 * * * \\
(0.0756)\end{array}$ \\
\hline Employees $>250$ & $\begin{array}{l}0.948 * * * \\
(0.143)\end{array}$ & $\begin{array}{l}0.941 \text { *** } \\
(0.142)\end{array}$ & $\begin{array}{l}0.942 * * * \\
(0.143)\end{array}$ \\
\hline \multicolumn{4}{|l|}{ Industry Effects } \\
\hline Country Effects & Yes & Yes & Yes \\
\hline Constant cut 1 & $\begin{array}{l}-0.315^{* *} \\
(0.150)\end{array}$ & $\begin{array}{l}-0.308^{* *} \\
(0.150)\end{array}$ & $\begin{array}{l}-0.309^{* *} \\
(0.150)\end{array}$ \\
\hline Constant cut 2 & $\begin{array}{l}0.513 * * * \\
(0.150)\end{array}$ & $\begin{array}{l}0.520 * * * \\
(0.150)\end{array}$ & $\begin{array}{l}0.520 * * * \\
(0.150)\end{array}$ \\
\hline Observations & 9236 & 9236 & 9236 \\
\hline $\mathrm{R}^{2}$ & 0.0889 & 0.0894 & 0.0894 \\
\hline$x^{2}$ & 819.7 & 830.0 & 831.0 \\
\hline
\end{tabular}

Robust standard errors in parentheses

*** $p<0.01 ; * * p<0.05 ; * p<0.1$ 
Table 12 Countries in the sample

\begin{tabular}{|c|c|}
\hline France & $4.20 \%$ \\
\hline Belgium & $3.04 \%$ \\
\hline The Netherlands & $3.20 \%$ \\
\hline Germany & $4.08 \%$ \\
\hline Italy & $3.70 \%$ \\
\hline Luxembourg & $1.60 \%$ \\
\hline Denmark & $3.70 \%$ \\
\hline Ireland & $2.15 \%$ \\
\hline United Kingdom & $3.62 \%$ \\
\hline Greece & $3.56 \%$ \\
\hline Spain & $4.06 \%$ \\
\hline Portugal & $2.84 \%$ \\
\hline Finland & $3.63 \%$ \\
\hline Sweden & $3.04 \%$ \\
\hline Austria & $2.92 \%$ \\
\hline Cyprus (Republic) & $1.61 \%$ \\
\hline Czech Republic & $3.10 \%$ \\
\hline Estonia & $2.94 \%$ \\
\hline Hungary & $3.20 \%$ \\
\hline Latvia & $2.90 \%$ \\
\hline Lithuania & $3.04 \%$ \\
\hline Malta & $1.57 \%$ \\
\hline Poland & $4.14 \%$ \\
\hline Slovakia & $3.65 \%$ \\
\hline Slovenia & $3.84 \%$ \\
\hline Bulgaria & $3.09 \%$ \\
\hline Romania & $3.12 \%$ \\
\hline Turkey & $2.58 \%$ \\
\hline Croatia & $1.68 \%$ \\
\hline Makedonia & $1.52 \%$ \\
\hline Montenegro & $0.83 \%$ \\
\hline Norway & $2.70 \%$ \\
\hline Iceland & $1.21 \%$ \\
\hline Israel & $1.88 \%$ \\
\hline USA & $2.04 \%$ \\
\hline$N$ & 9236 \\
\hline
\end{tabular}

Acknowledgements We are thankful to two anonymous reviewers for their constructive suggestions.

Open Access This article is distributed under the terms of the Creative Commons Attribution 4.0 International License (http:// creativecommons.org/licenses/by/4.0/), which permits unrestricted use, distribution, and reproduction in any medium, provided you give appropriate credit to the original author(s) and the source, provide a link to the Creative Commons license, and indicate if changes were made.

\section{References}

Acs, Z. J., \& Audretsch, D. B. (1990). Innovation and small firms. Cambridge: MIT Press.

Allouche, J. \& Laroche, P. (2006). The relationship between corporate social respon-sibility and corporate financial performance: A survey. Corporate social responsibility: Performance and stakeholders (pp. 3-40).

Balasubramanian, N., \& Lee, J. (2008). Firm age and innovation. Industrial and Corporate Change, 17(5), 1019-1047.

Bansal, P., \& Roth, K. (2000). Why companies go green: A model of ecological responsiveness. Academy of Management Journal, 43(4), 717-736.

Bhide, A. (1994). How entrepreneurs craft strategies that work. Harvard Business Review, 72(2), 150-161.

Busch, T., \& Hoffmann, V. H. (2011). How hot is your bottom line? Linking carbon and financial performance. Business \& Society, 50(2), 233-265.

Chen, P.H., Ong, C.F., \& Hsu, S.C. (2016). Understanding the relationships between environmental management practices and financial performances of multinational construction firms. Journal of Cleaner Production, 139, 750-760.

Coad, A., Frankish, J., Roberts, R. G., \& Storey, D. J. (2013). Growth paths and survival chances: an application of gambler's ruin theory. Journal of Business Venturing, 28(5), 615632.

Coad, A., Segarra, A., \& Teruel, M. (2016). Innovation and firm growth: does firm age play a role? Research Policy, 45(2), $387-400$.

Coase, R. H. (1960). The problem of social cost. Berlin: Springer.

Cohen, B., \& Winn, M. I. (2007). Market imperfections, opportunity and sustainable entrepreneurship. Journal of Business Venturing, 22(1), 29-49.

Cordeiro, J. J., \& Sarkis, J. (1997). Environmental proactivism and firm performance: evidence from security analyst earnings forecasts. Business Strategy and the Environ- ment, 6(2), 104-114.

Czarnitzki, D., \& Kraft, K. (2004). An empirical test of the asymmetric models on innovative activity: who invests more into R\&D, the incumbent or the challenger? Journal of Economic Behavior \& Organization, 54(2), 153-173.

Dean, T. J., \& McMullen, J. S. (2007). Toward a theory of sustainable entrepreneurship: reducing environmental degradation through entrepreneurial action. Journal of Business Venturing, 22(1), 50-76.

Delmas, M. A., Nairn-Birch, N., \& Lim, J. (2015). Dynamics of environmental and financial performance: the case of greenhouse gas emissions. Organization \& Environ- ment, 28(4), 374-393.

Djupdal, K., \& Westhead, P. (2015). Environmental certification as a buffer against the liabilities of newness and smallness: firm performance benefits. International Small Business Journal, 33(2), 148-168.

Dowell, G., Hart, S., \& Yeung, B. (2000). Do corporate global environmental standards create or destroy market value? Management Science, 46(8), 1059-1074.

Esty, D., \& Winston, A. (2009). Green to gold: How smart companies use environmental strategy to innovate, create value, and build competitive advantage. Hoboken: John Wiley \& Sons. 
European Commission, Brussels (2014) Flash Eurobarometer 342 (Small and Medium Enterprises, Resource Efficiency and Green Markets, wave 1). TNS Political \& Social [producer]. GESIS Data Archive, Cologne. ZA5777 Data file Version 1.0.0. https://doi.org/10.4232/1.11879.

Ford, J. A., Steen, J., \& Verreynne, M.-L. (2014). How environmental regulations affect innovation in the Australian oil and gas industry: going beyond the Porter hypothesis. Journal of Cleaner Production, 84, 204-213.

Gray, W. B. \& Shadbegian, R. J. (1993). Environmental regulation and manufacturing productivity at the plant level (No. w4321). Cambridge: National Bureau of Economic Research.

Hamschmidt, J., \& Dyllick, T. (2001). Iso 14001. Greener Management International, 2001(34), 43-54.

Hart, S. L. (1995). A natural-resource-based view of the firm. Academy of Management Review, 20(4), 986-1014.

Hart, S. L., \& Ahuja, G. (1996). Does it pay to be green? An empirical examination of the relationship between emission reduction and firm performance. Business Strategy and the Environment, 5(1), 30-37.

Hockerts, K. (2006). Cafédirect: a social entrepreneurial fair trade success. In The new social entrepreneurship, what awaits social entrepreneurial ventures. Cheltenham: Edward Elgar.

Hockerts, K., \& Wustenhagen, R. (2010). Greening goliaths versus emerging davids: theorizing about the role of incumbents and new entrants in sustainable entrepreneurship. Journal of Business Venturing, 25(5), 481-492.

Huergo, E., \& Jaumandreu, J. (2004). How does probability of innovation change with firm age? Small Business Economics, 22(3-4), 193-207.

King, A. A., \& Lenox, M. J. (2001). Does it really pay to be green? An empirical study of firm environmental and financial performance: an empirical study of firm environmental and financial performance. Journal of Industrial Ecology, 5(1), 105-116.

Klassen, R. D., \& McLaughlin, C. P. (1996). The impact of environmental management on firm performance. Management Science, 42(8), 1199-1214.

Klassen, R. D., \& Whybark, D. C. (1999). The impact of environmental technologies on manufacturing performance. Academy of Management Journal, 42(6), 599-615.

Klewitz, J., \& Hansen, E. G. (2014). Sustainability-oriented innovation of smes: a systematic review. Journal of Cleaner Production, 65, 57-75.

Lannelongue, G., Gonzalez-Benito, J., \& Gonzalez-Benito, O. (2015). Input, output, and environmental management productivity: effects on firm performance. Business Strategy and the Environment, 24(3), 145-158.

Margolis, J. D., \& Walsh, J. P. (2001). People and profi the search for a link between a company's social and fi performance. Hove: Psychology Press.
Nehrt, C. (1996). Timing and intensity effects of environmental investments. Strategic Management Journal, 17(7), 535547.

Nicholls, A., \& Opal, C. (2005). Fair trade: Market-driven ethical consumption. Thousand Oaks: Sage.

Nishitani, K. (2011). An empirical analysis of the effects on firms' economic performance of implementing environmental management systems. Environmental and Resource Economics, 48(4), 569-586.

Orlitzky, M., Schmidt, F. L., \& Rynes, S. L. (2003). Corporate social and financial performance: a meta-analysis. Organization Studies, 24(3), 403-441.

Porter, M. E., \& Van der Linde, C. (1995). Toward a new conception of the environment-competitiveness relationship. The Journal of Economic Perspectives, 9(4), 97-118.

Pujari, D. (2006). Eco-innovation and new product development: understanding the influence on market performance. Technovation, 26(1), 76-85.

Reinhardt, F. L. (1998). Environmental product differentiation: Implications for corporate strategy. California Management Review, 40(4), 43-73.

Sarkis, J., \& Cordeiro, J. J. (2001). An empirical evaluation of environmental efficiencies and firm performance: pollution prevention versus end-of-pipe practice. European Joural of Operational Research, 135(1), 102-113.

Schaltegger, S. (2002). A framework for ecopreneurship. Greener Management Interna- tional, 2002(38), 45-58.

Sharma, S., \& Vredenburg, H. (1998). Proactive corporate environmental strategy and the development of competitively valuable organizational capabilities. Strategic Management Journal, 19(8), 729-753.

Stefan, A., \& Paul, L. (2008). Does it pay to be green? A systematic overview. The Academy of Management Perspectives, 22(4), 45-62.

Stenzel, T., \& Frenzel, A. (2008). Regulating technological change-The strategic reactions of utility companies towards subsidy policies in the German, Spanish and UK electricity markets. Energy Policy, 36(7), 2645-2657.

Stinchcombe, A. L. (1965). Organizations and social structure. Handbook of organizations, 44(2), 142-193.

Temminck, E., Mearns, K., \& Fruhen, L. (2015). Motivating employees towards sustainable behaviour. Business Strategy and the Environment, 24(6), 402-412.

Walley, N., \& Whitehead, B. (1994). It's not easy being green. Reader in Business and the Environment, 36, 81.

Wüstenhagen, R., Markard, J., \& Truffer, B. (2003). Diffusion of green power products in switzerland. Energy Policy, 31(7), 621-632.

York, J. G., \& Venkataraman, S. (2010). The entrepreneurenvironment nexus: uncertainty, innovation, and allocation. Journal of Business Venturing, 25(5), 449-463. 